\title{
Wrinklefree and deadly: BoNTs
}

DOI:

10.1038/nrn2085

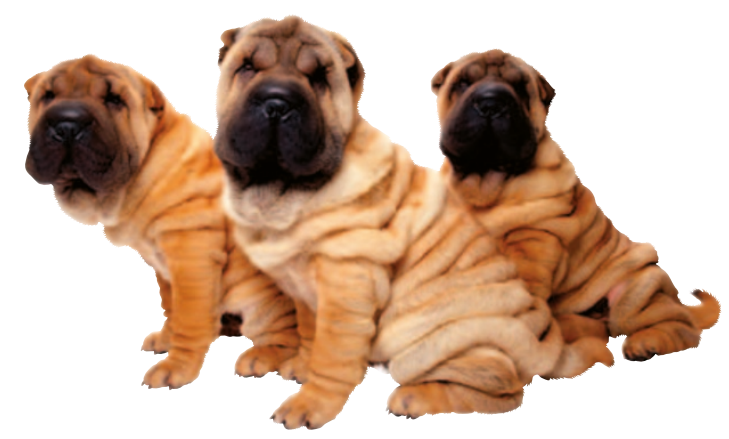

cleave core proteins of the vesicle fusion machinery, leading to inhibition of neurotransmission at the neuromuscular junction, causing muscle paralysis.

Membrane binding of BoNTs involves interaction with protein receptors and gangliosides, which are complex glycolipids abundant on the surface of nerve cells. For BoNT/B and BoNT/G, the protein receptors are the luminal domains of synaptotagmin (SYT) I and II, which are only exposed to the cell surface after vesicle fusion with the plasma membrane. Now different research groups have analysed the structural and molecular nature of these interactions.

In two papers published in Nature, the authors solved the crystal structures of BoNT/B bound to the SYTII recognition domain, and of a fusion protein composed of the cell binding domain of BoNT/B and the entire luminal domain of SYTII, respectively. These studies showed that SYTII binds to the most distal part of BoNT/B, adjacent to the binding pocket for gangliosides. This binding induces the formation of an $\alpha$-helix in the SYTII binding domain. The residues of BoNT/B and SYTII that are crucial for the toxin-receptor interaction as revealed by the crystal structure were mutated. Jin et al. and Rummel et al. systematically studied the binding properties of these mutants by in vitro pull-down assays and the toxicity of the BoNT mutants in phrenic nerve preparations of wild-type and ganglioside deficient mice, which is an established method for assaying toxicity. The mutants which showed lower binding affinity in the pull-down assays also displayed attenuated toxicity.

Together, these findings identify key residues in the binding domains of BoNT/B and G and SYTI and II. The identification of the BoNT/ B-SYTII interface can now be used as a target for the development of preventative vaccines or inhibitors. Already, the crystal structures of similar complexes have been used to explain the effects of neutralizing therapeutic antibodies against other BoNTs, as described in a related Nature Biotechnology structure paper by Garcia-Rodriguez et al. Designing mutated versions of BoNT could also be used to target different cell types and to exploit BoNTs as drug delivery systems.

Claudia Wiedemann

\section{ORIGINAL RESEARCH PAPERS}

Rummel, A. et al. Identification of the protein receptor binding site of botulinum neurotoxins $B$ and $\mathrm{G}$ proves the double-receptor concept. PNAS 104, 359-364 (2007) | Jin, R. et al. Botulinum neurotoxin $B$ recognizes its protein receptor with high affinity and specificity. Nature $\mathbf{4 4 4}$, 1092-1095 (2006) | Chai, Q. et al. Structural basis of cell surface receptor recognition by botulinum neurotoxin B. Nature 444, 1096-1100 (2006) | Garcia-Rodriguez, C. et al. Molecular evolution of antibody cross-reactivity for two subtypes of type A botulinum neurotoxin. Nature Biotech. 25, 107-116 (2007) 